\title{
Finite-Time Performance Guaranteed Event-Triggered Adaptive Control for Nonlinear Systems with Unknown Control Direction
}

\author{
Min Wang · Lixue Wang
}

Received: date / Accepted: date

\begin{abstract}
This paper studies the issue of finite-time performance guaranteed event-triggered (ET) adaptive neural tracking control for strict-feedback nonlinear systems with unknown control direction. A novel finitetime performance function is first constructed to describe the prescribed tracking performance, and then a new lemma is given to show the differentiability and boundedness for the performance function, which is important for the verification of the closed-loop stability. Furthermore, with the help of the error transformation technique, the origin constrained tracking error is transformed into an equivalent unconstrained one. By utilizing the first-order sliding mode differentiator, the issue of "explosion of complexity" caused by the backstepping design is adequately addressed. Subsequently, an ingenious adaptive updated law is given to co-design the controller and the ET mechanism by the combination of the Nussbaum-type function, thus effectively handling the influences of the measurement error resulted from the ET mechanism and the challenge of the controller design caused by the unknown control direction. The presented event-triggered control scheme can not only guarantee the prescribed tracking performance, but also alleviate the communication burden simultaneously. Finally, numerical and practical examples are provided to demonstrate the validity of the proposed control strategy.
\end{abstract}

\section{Wang, L. Wang}

School of Automation Science and Engineering, Guangdong Provincial Key Laboratory of Technique and Equipment for Macromolecular Advanced Manufacturing, South China University of Technology, Guangzhou, 510641, China.

Tel.: +8620-87111258

Fax: +8620-87111258

E-mail: auwangmin@scut.edu.cn
Keywords Event-triggered control · Finite-time prescribed performancel · First-order sliding mode differentiator · Strict-feedback systems · Unknown control direction

\section{Introduction}

In the past decades, strict-feedback nonlinear systems (SFNSs), as a special kind of nonlinear systems, have evoked widespread attention because of their powerful capability to model various kinds of practical systems, such as chemical stirred tank reactor [1], flexible joint robotic system [2], hypersonic flight vehicles [3] and so on. As a consequence, for the control problems of SFNSs, many scholars have carried out in-depth research owing to the huge needs in engineering applications. The adaptive backstepping method [4], as a breakthrough method in the field of nonlinear control, has become an effective way to deal with the control issues of SFNSs. With the aid of neural network (NN) [5] or fuzzy logic approximator, the adaptive backstepping method has been widely used to control the SFNSs with unknown nonlinear dynamics [6-9]. However, the results mentioned above exist the issue of "explosion of complexity" resulted from repeated derivations of the virtual controller in backstepping design. To handle such a problem, many useful approaches have been developed. For example, the dynamic surface control (DSC) method was employed to estimate the derivative of the virtual controller in $[10,11]$. The authors in [12] use a more efficient technique namely first-order sliding mode differentiator (FOSMD) to avert tedious calculations.

Noting that in some practical systems, the tracking error often needs to meet some specified transient 
and steady state performance indicators, such as small overshoot, fast convergence speed and small steadystate error, so as to ensure the control performance of the system. In view of such situation, the authors in [13] firstly presented a performance function transformation method, whose basic idea was to convert the constrained tracking error into the unconstrained one. Subsequently, such a method was not only applied to more general systems with miscellaneous engineeringoriented phenomena, such as unmodeled dynamics [14], input saturation [15], actuator failures [16] and input quantization [17], but also widely employed to a great deal of practical systems $[18,19]$. Unfortunately, the performance functions in the aforementioned works are not concerned about finite time convergence, which limits their engineering applications with high-accuracy control. The prescribed finite time convergence issue is extremely difficult for controller design of nonlinear systems. More recently, this issue were gained some concern, and a few preliminary and valuable results were developed in [20-24]. Specifically, the authors in [20] constructed a finite-time performance function, whose convergence time can be set arbitrarily. This result [20] has been extended to a finite-time fuzzy tracking control scheme for SFNSs with dynamic disturbances [22]. And then, the finite-time consensus tracking control was handled in [24] for multi agent systems with prescribed performance and mismatched uncertainties.

It is worth pointing out that the results mentioned above can only be obtained when the control direction of the system is known in advance. Once the control direction is unknown, these control schemes are no longer feasible. In view of such a case, the authors in [25] firstly developed a Nussbaum-type function (NTF), which effectively handled the difficulty in controller design caused by the unknown control direction. Subsequently, on the basis of NTF, a great number of interesting works have been reported. To mention a few, in combination with NTF and Barrier Lyapunov function, an adaptive tracking control strategy has been constructed for a class of state-constrained SFNSs with unknown control direction [26]. Based on the NTF and fuzzy logic approximator, the DSC fuzzy controller presented in [27] has successfully guaranteed the stability of uncertain non-strict-feedback systems with unknown virtual control coefficients. These NTF-based results does not concern two hot directions: the prescribed finite time convergence and the limited network resources.

Recently, networked control has been developed rapidly due to its peculiarities of low cost, good flexibility, reliable operation, convenient installation, etc. However, the network bandwidth is limited, which inevitably brings some problems including transmission delay [28], packet disorder [29], and so on. To deal with such problems, the authors in [30] presented an event-triggered control (ETC) approach, which efficaciously economizes the network resource. On the basis of the ETC, a series of valuable results have been reported [31-37]. For instance, a relative threshold ETC method has been developed for SFNSs with unknown parameters in [33], which achieves a good balance between the system performance and the limited network resources. In combination with the relative threshold strategy, some fruitful ETC schemes have been presented for more general nonlinear systems with prescribed performance [34], input saturation [36] and unmeasured state [37]. Unfortunately, these aforementioned ETC methods can not be directly extended to prescribed performanceguaranteed SFNSs with unknown control direction. The main reason lies in the unknown control direction is coupled in the compensation process of the measurement error between the controller and the actuator. In this case, it becomes significantly challenging to construct a suitable ETC scheme to weaken the effect of the measurement error on system stability. As a consequence, it is significant and necessary to investigate the ETC for SFNSs subject to both prescribed performance and unknown control direction.

According to the aforementioned discussion so far, the main objective of this paper is to construct a finitetime performance guaranteed ETC scheme for SFNSs with unknown control direction. Firstly, the finite-time performance function is constructed to guarantee the tracking performance constraint by the aid of the error transformation approach. Secondly, the FOSMD is embedded in the backstepping procedure to cope with the issue of "explosion of complexity", and then an ingenious adaptive law is given to facilitate the co-design of controller and ET mechanism. Finally, a finite-time performance guaranteed ETC scheme is developed based on the novel adaptive law and the NTF, which guarantees the prescribed tracking performance, alleviates the communication burden and compensates the measurement error at the same time. The main contributions are listed as follows:

1) A novel finite-time performance function is developed such that the prescribed tracking performance is achieved in a predetermined finite time instead of the infinite time. Moreover, a new lemma is derived to show the differentiability and boundedness of the constructed performance function, which plays an important role for the system stability;

2) A novel adaptive law is given to estimate the upper bound of the actual control gain, and then the controller and the ET mechanism are co-designed 
to compensate successfully the measurement error resulted from the ET mechanism;

3) In combination with such an adaptive law and the hyperbolic tangent function, a novel ET actuator under the relative threshold strategy is proposed, thereby achieving the prescribed finite-time tracking performance and saving the communication resource.

\section{Problem formulation and preliminaries}

\subsection{System description}

This paper considers a class of SFNSs as follows:

$$
\left\{\begin{array}{l}
\dot{x}_{i}=f_{i}\left(\underline{x}_{i}\right)+g_{i}\left(\underline{x}_{i}\right) x_{i+1}, i=1,2, \ldots, n-1 \\
\dot{x}_{n}=f_{n}\left(\underline{x}_{n}\right)+g_{n}\left(\underline{x}_{n}\right) u \\
y=x_{1}
\end{array}\right.
$$

where $\underline{x}_{i}=\left[x_{1}, x_{2}, \ldots, x_{i}\right]^{T} \in \mathbb{R}^{i}(i=1,2, \ldots, n)$, $u \in \mathbb{R}$ and $y \in \mathbb{R}$ denote, respectively, the state vector, the system control input and output. $f_{i}\left(\underline{x}_{i}\right)$ and $g_{i}\left(\underline{x}_{i}\right)$ stand for the unknown smooth nonlinear functions.

Assumption 1 [38] The control coefficients $g_{i}\left(\underline{x}_{i}\right), i=$ $1,2, \ldots, n$ with unknown signs and $g_{i}\left(\underline{x}_{i}\right) \neq 0$. Moreover, $g_{i}\left(\underline{x}_{i}\right)$ satisfy $g_{i 1} \leq\left|g_{i}\left(\underline{x}_{i}\right)\right| \leq g_{i 2}$, where $g_{i 1}$ and $g_{i 2}$ are unknown positive constants.

Assumption 2 The reference trajectory $y_{d}$ and its time derivatives $\dot{y}_{d}$ and $\ddot{y}_{d}$ are bounded.

\subsection{Radial basis function NNs}

It has been shown in [39] that the radial basis function (RBF) NN has a powerful ability to approximate any unknown smooth nonlinear function $T(Z)$ over a compact set $\Omega_{Z}$ as

$T(Z)=W^{* T} S(Z)+\delta(Z)$

where $Z \in \Omega_{Z} \subset \mathbb{R}^{o}$ sands for the input vector of NN, $S(Z)=\left[S_{1}(Z), \ldots, S_{l}(Z)\right]^{T} \in \mathbb{R}^{l}$ denotes the Gaussian basis function vector, where $S_{i}(Z)=\exp [-(Z-$ $\left.\left.\Theta_{i}\right)^{T}\left(Z-\Theta_{i}\right) / \triangle_{i}\right]$ with $\Theta_{i}=\left[\Theta_{i 1}, \ldots, \Theta_{i o}\right]^{T}$ and $\triangle_{i}$ are the center and width of NN, respectively. $W^{*} \in \mathbb{R}^{l}$ represents the optimal neural weight vector with $l>1$ being the number of neural node, $\delta(Z)$ denotes the approximation error satisfying $|\delta(Z)| \leq \bar{\delta}$, where $\bar{\delta}>0$ is an arbitrarily small constant.
2.3 Novel finite-time performance function

A novel finite-time performance function is constructed as follows:

$\phi(t)=\left\{\begin{array}{lc}-\tanh \left(\phi_{1}+\frac{t}{T_{c}-t}\right)+\phi_{2}+1,0 \leq t<T_{c} \\ \phi_{2}, & t>T_{c}\end{array}\right.$

where $\phi_{1}, \phi_{2}$ and $T_{c}$ are positive constants, $\tanh (\cdot)$ represents the hyperbolic tangent function.

Lemma $1 \phi(t)$ and $\dot{\phi}(t)$ are continuously differentiable and bounded on $[0,+\infty)$ and $\ddot{\phi}(t)$ is continuous and bounded on $[0,+\infty)$.

Proof: See the Appendix.

Remark 1 Notice that the finite-time performance control problem has been considered in the existing results in [20-22], [24]. These results mentioned above require some restrictions. For example, the initial condition $\phi(0)$ is required to satisfy $\phi(0) \leq 1$ in [20-22]; and $\phi(t)$ depends on the order $n$ of the controlled system [24], which makes the computational complexity of $\phi(t)$ greatly increase for high-order nonlinear systems. Compared with the existing works [20-22], [24], it is obvious from (2) that the finite-time performance function developed in this paper is easy-to-implement due to the mild initial condition $\phi(0)=-\tanh \left(\phi_{1}\right)+\phi_{2}+1>0$ and the independence of system order $n$.

In this paper, the tracking error $e_{1}=y-y_{d}$ should remain within the predefined performance constraint as follows

$-\varsigma_{1} \phi(t)<e_{1}(t)<\varsigma_{2} \phi(t)$

where $\varsigma_{1}$ and $\varsigma_{2}$ are both positive design parameters.

It can be concluded from $(2)$ and $(3)$ that $-\varsigma_{1} \phi(0)$ and $\varsigma_{2} \phi(0)$ with $\phi(0)=-\tanh \left(\phi_{1}\right)+\phi_{2}+1$ denote, respectively, the minimum value of the transient undershoot and the maximum value of the transient overshoot of $e_{1} .-\varsigma_{1} \phi_{2}$ and $\varsigma_{2} \phi_{2}$ represent the low bound and upper bound of the steady-state tracking error $e_{1}$, respectively. Besides, $T_{c}$ stands for the time when tracking error $e_{1}$ decays to the steady-state value $\phi_{2}$.

\subsection{Useful Definition and Lemmas}

Definition 1 [25] Any smooth even function $N(\varphi)$ can be called as a function of Nussbaum-type when it satisfies $\lim _{s \rightarrow \infty} \sup \frac{1}{s} \int_{0}^{s} N(\varphi) d \varphi=+\infty$ and $\lim _{s \rightarrow \infty} \inf \frac{1}{s} \int_{0}^{s}$ $N(\varphi) d \varphi=-\infty$. 
Lemma 2 [25] Let $V(t) \geq 0$ and $\varphi(t)$ be smooth functions defined on $\left[0, t_{f}\right)$, and $N(\varphi)=\varphi^{2} \cos (\varphi)$. For $\forall t \in\left[0, t_{f}\right)$, if

$V(t) \leq r_{1}+e^{-r_{2} t} \int_{0}^{t}[g(x(\tau)) N(\varphi)+1] \dot{\varphi} e^{r_{2} \tau} d \tau$

where $r_{1}$ and $r_{2}$ stand for, respectively, a suitable constant and a positive constant. $g(x(t))$ denotes a unknown smooth function which takes values in the unknown closed interval $D=\left[d^{-}, d^{+}\right]$with $0 \notin D$. Then, $V(t), \int_{0}^{t} g(x(\tau)) N(\varphi) \dot{\varphi} d \tau$ and $\varphi(t)$ must be bounded on $\left[0, t_{f}\right)$.

The FOSMD [40-42] is described as

$$
\left\{\begin{array}{l}
\dot{\vartheta}_{1}=\varrho_{1} \\
\varrho_{1}=-\omega_{1}\left|\vartheta_{1}-v\right|^{\frac{1}{2}} \operatorname{sign}\left(\vartheta_{1}-v\right)+\vartheta_{2} \\
\dot{\vartheta}_{2}=-\omega_{2} \operatorname{sign}\left(\vartheta_{2}-\varrho_{1}\right)
\end{array}\right.
$$

where $\vartheta_{1}, \vartheta_{2}$ and $\varrho_{1}$ represent the system states, $\omega_{1}$ and $\omega_{2}$ stand for the positive design constants, $v$ denotes the input signal of the FOSMD. Then, we can obtain the following lemmas.

Lemma 3 [41, 42] By selecting suitable design constants, the following equalities can be obtained after a finite time in the absence of input noises:

$\vartheta_{1}=v_{0}, \varrho_{1}=\dot{v}_{0}$

Notice that Lemma 3 is derived under the case of no input noises, i.e. $v=v_{0}$, when the input noise exists, the following Lemma holds.

Lemma 4 [41,42] If the input noise meets $\left|v-v_{0}\right| \leq$ $\iota$, the following inequalities can be obtained in a finite time:

$$
\begin{aligned}
& \left|\vartheta_{1}-v_{0}\right| \leq a_{1} \iota=\bar{\ell} \\
& \left|\varrho_{1}-\dot{v}_{0}\right| \leq b_{1} \iota^{\frac{1}{2}}=\bar{\varepsilon}
\end{aligned}
$$

where $\bar{\ell}$ and $\bar{\varepsilon}$ are both positive constants exclusively depended on the design constants of the FOSMD.

Lemma 5 [43] For any $\pi>0$ and $\hbar \in \mathbb{R}$, the inequality $0<|\hbar|-\hbar \tanh (\hbar / \pi) \leq 0.2785 \pi$ holds.

\section{Event-triggered adaptive NN controller design}

Define the following error variables:

$z_{i}=x_{i}-\alpha_{i-1}, i=2,3, \ldots, n$

where virtual law $\alpha_{j}(j=1,2, \ldots, n-1)$ will be designed later.
Step 1: For the purpose of transforming the constrained tracking error $e_{1}(3)$ into the equivalent unconstrained variable $z_{1}$, we introduce a smooth and strictly increasing transformation function $\Upsilon\left(z_{1}\right)$, which satisfies

$\left\{\begin{array}{l}-\varsigma_{1}<\Upsilon\left(z_{1}\right)<\varsigma_{2} \\ \lim _{z_{1} \rightarrow+\infty} \Upsilon\left(z_{1}\right)=\varsigma_{2}, \lim _{z_{1} \rightarrow-\infty} \Upsilon\left(z_{1}\right)=-\varsigma_{1} .\end{array}\right.$

With the help of $\Upsilon\left(z_{1}\right)$, (3) can be expressed as

$e_{1}(t)=\phi(t) \Upsilon\left(z_{1}\right)$

where $\Upsilon\left(z_{1}\right)$ is constructed as follows

$\Upsilon\left(z_{1}\right)=\frac{\varsigma_{2} e^{z_{1}}-\varsigma_{1} e^{-z_{1}}}{e^{z_{1}}+e^{-z_{1}}}$.

Then, it follows from (6) that

$z_{1}=\Upsilon^{-1}\left(\frac{e_{1}(t)}{\phi(t)}\right)=\frac{1}{2} \ln \left(\frac{\varsigma_{1}+e_{1}(t) / \phi(t)}{\varsigma_{2}-e_{1}(t) / \phi(t)}\right)$.

Based on (1) and (7), one obtains

$$
\begin{aligned}
\dot{z}_{1} & =\xi\left[\dot{e}_{1}(t)-e_{1}(t) \frac{\dot{\phi}(t)}{\phi(t)}\right] \\
& =\xi\left[f_{1}\left(x_{1}\right)+g_{1}\left(x_{1}\right) x_{2}-\dot{y}_{d}-e_{1}(t) \frac{\dot{\phi}(t)}{\phi(t)}\right]
\end{aligned}
$$

where $\xi=(1 / 2 \phi(t))\left[1 /\left(\varsigma_{1}+e_{1}(t) / \phi(t)\right)+1 /\left(\varsigma_{2}-e_{1}(t) / \phi(t)\right)\right]$. Moreover, by (3), it can be concluded that $\xi>0$.

Define the Lyapunov function candidate as follows

$V_{1}=\frac{1}{2} z_{1}^{2}+\frac{1}{2} \tilde{W}_{1} \Gamma_{1}^{-1} \tilde{W}_{1}$

where $\Gamma_{1}=\Gamma_{1}^{T}>0$ is a constant matrix, $\tilde{W}_{1}=\hat{W}_{1}-$ $W_{1}^{*}$ stands for the weight estimation error with $\hat{W}_{1}$ being the estimation of $W_{1}^{*}$. Then, the dynamic of $V_{1}$ along (8) is

$$
\begin{aligned}
\dot{V}_{1}= & z_{1} \xi\left[g_{1}\left(x_{1}\right) x_{2}+f_{1}\left(x_{1}\right)-\dot{y}_{d}-e_{1}(t) \frac{\dot{\phi}(t)}{\phi(t)}\right] \\
& +\tilde{W}_{1}^{T} \Gamma_{1}^{-1} \dot{\hat{W}}_{1} .
\end{aligned}
$$

Let unknown function

$T_{1}\left(Z_{1}\right)=\xi\left[f_{1}\left(x_{1}\right)-\dot{y}_{d}\right]$

where $Z_{1}=\left[x_{1}, y_{d}, \dot{y}_{d}, \phi(t)\right]^{T} \in \mathbb{R}^{4}$.

We utilize the RBF NN to approximate $T_{1}\left(Z_{1}\right)$ as

$T_{1}\left(Z_{1}\right)=W_{1}^{* T} S_{1}\left(Z_{1}\right)+\delta_{1}\left(Z_{1}\right)$

where $\left|\delta_{1}\left(Z_{1}\right)\right| \leq \bar{\delta}_{1}$ 
By substituting (11) into (10) yields

$$
\begin{aligned}
\dot{V}_{1}= & z_{1} \xi\left[g_{1}\left(x_{1}\right) x_{2}-e_{1}(t) \frac{\dot{\phi}(t)}{\phi(t)}\right]+z_{1} W_{1}^{* T} S_{1}\left(Z_{1}\right) \\
& +z_{1} \delta_{1}\left(Z_{1}\right)+\tilde{W}_{1}^{T} \Gamma_{1}^{-1} \dot{\hat{W}}_{1}
\end{aligned}
$$

According to Young's inequality, we have

$$
z_{1} \delta_{1}\left(Z_{1}\right) \leq \frac{\lambda_{1}}{2} z_{1}^{2}+\frac{1}{2 \lambda_{1}} \bar{\delta}_{1}^{2}
$$

where $\lambda_{1}>0$ is a design constant.

Substituting (13) into (12), one has

$$
\begin{aligned}
\dot{V}_{1} \leq & z_{1} \xi\left[g_{1}\left(x_{1}\right) x_{2}-e_{1}(t) \frac{\dot{\phi}(t)}{\phi(t)}\right]+z_{1} W_{1}^{* T} S_{1}\left(Z_{1}\right) \\
& +\tilde{W}_{1}^{T} \Gamma_{1}^{-1} \dot{\hat{W}}_{1}+\frac{\lambda_{1}}{2} z_{1}^{2}+\frac{1}{2 \lambda_{1}} \bar{\delta}_{1}^{2}
\end{aligned}
$$

The virtual law $\alpha_{1}$ and neural weight updated law $\dot{\hat{W}}_{1}$ are constructed as follows

$$
\begin{aligned}
\alpha_{1}= & \frac{1}{\xi} N\left(\varphi_{1}\right) \psi_{1} \\
\psi_{1}= & k_{1} z_{1}+\hat{W}_{1}^{T} S_{1}\left(Z_{1}\right)+\left(\frac{\lambda_{1}}{2}+\frac{\xi^{2}}{2}\right) z_{1} \\
& -\xi e_{1}(t) \frac{\dot{\phi}(t)}{\phi(t)} \\
\dot{\varphi}_{1}= & z_{1} \psi_{1} \\
\dot{\hat{W}}_{1}= & \Gamma_{1}\left[z_{1} S_{1}\left(Z_{1}\right)-\sigma_{1} \hat{W}_{1}\right]
\end{aligned}
$$

where $k_{1}>0$ and $\sigma_{1}>0$ are both design parameters.

Based on (15)-(17) and $x_{2}=z_{2}+\alpha_{1}$, we obtain

$$
\begin{aligned}
z_{1} \xi g_{1}\left(x_{1}\right) x_{2}= & z_{1} \xi\left[g_{1}\left(x_{1}\right) z_{2}+e_{1}(t) \frac{\dot{\phi}(t)}{\phi(t)}\right]-\left(k_{1}\right. \\
& \left.+\frac{\lambda_{1}}{2}+\frac{\xi^{2}}{2}\right) z_{1}^{2}-z_{1} \hat{W}_{1}^{T} S_{1}\left(Z_{1}\right) \\
& +\left(g_{1}\left(x_{1}\right) N\left(\varphi_{1}\right)+1\right) \dot{\varphi}_{1} .
\end{aligned}
$$

Substituting (18) and (19) into (14), one can obtain

$$
\begin{aligned}
\dot{V}_{1} \leq & z_{1} \xi g_{1}\left(x_{1}\right) z_{2}+\left(g_{1}\left(x_{1}\right) N\left(\varphi_{1}\right)+1\right) \dot{\varphi}_{1} \\
& -\left(k_{1}+\frac{\xi^{2}}{2}\right) z_{1}^{2}-\sigma_{1} \tilde{W}_{1}^{T} \hat{W}_{1}+\frac{1}{2 \lambda_{1}} \bar{\delta}_{1}^{2} .
\end{aligned}
$$

With the help of Young's inequality, we have

$$
\begin{aligned}
& z_{1} \xi g_{1}\left(x_{1}\right) z_{2} \leq \frac{\xi^{2}}{2} z_{1}^{2}+\frac{1}{2} g_{1}^{2}\left(x_{1}\right) z_{2}^{2} \\
& -\sigma_{1} \tilde{W}_{1}^{T} \hat{W}_{1} \leq-\frac{\sigma_{1}}{2}\left\|\tilde{W}_{1}\right\|^{2}+\frac{\sigma_{1}}{2}\left\|W_{1}^{*}\right\|^{2} .
\end{aligned}
$$

Substituting (21) into (20), one has

$$
\begin{aligned}
\dot{V}_{1} \leq & -k_{1} z_{1}^{2}-\frac{\sigma_{1}}{2}\left\|\tilde{W}_{1}\right\|^{2}+\frac{\sigma_{1}}{2}\left\|W_{1}^{*}\right\|^{2}+\frac{1}{2 \lambda_{1}} \bar{\delta}_{1}^{2} \\
& +\left(g_{1}\left(x_{1}\right) N\left(\varphi_{1}\right)+1\right) \dot{\varphi}_{1}+\frac{1}{2} g_{1}^{2}\left(x_{1}\right) z_{2}^{2} \\
\leq & -p_{1} V_{1}+q_{1}+\left(g_{1}\left(x_{1}\right) N\left(\varphi_{1}\right)+1\right) \dot{\varphi}_{1} \\
& +\frac{1}{2} g_{1}^{2}\left(x_{1}\right) z_{2}^{2}
\end{aligned}
$$

where $q_{1}=\sigma_{1}\left\|W_{1}^{*}\right\|^{2} / 2+\bar{\delta}_{1}^{2} / 2 \lambda_{1}$ and $p_{1}=\min \left\{2 k_{1}\right.$, $\left.\sigma_{1} / \lambda_{\max }\left(\Gamma_{1}^{-1}\right)\right\}$.

Step $i(2 \leq i \leq n-1)$ : Noting that $z_{i}=x_{i}-\alpha_{i-1}$, its derivative along (1) is

$\dot{z}_{i}=f_{i}\left(\underline{x}_{i}\right)+g_{i}\left(\underline{x}_{i}\right) x_{i+1}-\dot{\alpha}_{i-1}$.

In order to effectively estimate $\dot{\alpha}_{i-1}$ and overcome the issue of explosion of complexity, the FOSMD is constructed as

$$
\left\{\begin{array}{l}
\dot{\vartheta}_{i 1}=\varrho_{i 1} \\
\varrho_{i 1}=-\omega_{i 1}\left|\vartheta_{i 1}-\alpha_{i-1}\right|^{\frac{1}{2}} \operatorname{sign}\left(\vartheta_{i 1}-\alpha_{i-1}\right)+\vartheta_{i 2} \\
\dot{\vartheta}_{i 2}=-\omega_{i 2} \operatorname{sign}\left(\vartheta_{i 2}-\varrho_{i 1}\right)
\end{array}\right.
$$

where $\vartheta_{i 1}, \vartheta_{i 2}$ and $\varrho_{i 1}$ represent the system states, $\omega_{i 1}$ and $\omega_{i 2}$ are both positive design constants.

Combining with (24) and Lemma 3-4, it follows that

$\dot{\alpha}_{i-1}=\varrho_{i 1}+\varepsilon_{i}$

where $\varepsilon_{i}$ satisfies $\left|\varepsilon_{i}\right| \leq \bar{\varepsilon}_{i}$ with $\bar{\varepsilon}_{i}$ being a positive constant. If the input signal $\alpha_{i-1}$ of the FOSMD (24) is not influenced by the noise, it can be concluded from Lemma 3 that $\bar{\varepsilon}_{i}=0$. Moreover, if the input signal $\alpha_{i-1}$ is influenced by the bounded noise, we can conclude from Lemma 4 that $\left|\varepsilon_{i}\right| \leq \bar{\varepsilon}_{i}$.

Consider the Lyapunov function candidate

$V_{i}=\frac{1}{2} z_{i}^{2}+\frac{1}{2} \tilde{W}_{i}^{T} \Gamma_{i}^{-1} \tilde{W}_{i}$

where $\Gamma_{i}=\Gamma_{i}^{T}>0$ is a constant matrix, $\tilde{W}_{i}=\hat{W}_{i}-W_{i}^{*}$ stands for the weight estimation error with $\hat{W}_{i}$ being the estimation of $W_{i}^{*}$.

Based on (23), we have

$$
\begin{aligned}
\dot{V}_{i}= & z_{i}\left[g_{i}\left(\underline{x}_{i}\right) x_{i+1}+W_{i}^{* T} S_{i}\left(Z_{i}\right)+\delta_{i}\left(Z_{i}\right)-\dot{\alpha}_{i-1}\right] \\
& +\tilde{W}_{i}^{T} \Gamma_{i}^{-1} \dot{\hat{W}}_{i}
\end{aligned}
$$

where $W_{i}^{* T} S_{i}\left(Z_{i}\right)$ is employed to approximate $f_{i}\left(\underline{x}_{i}\right)$ with $Z_{i}=\left[x_{1}, x_{2}, \ldots, x_{i}\right]^{T} \in \mathbb{R}^{i}, W_{i}^{*}$ denotes the ideal weights vector and $\left|\delta_{i}\left(Z_{i}\right)\right| \leq \bar{\delta}_{i}$.

According to Young's inequality, one can obtain

$z_{i} \delta_{i}\left(Z_{i}\right) \leq \frac{\lambda_{i}}{2} z_{i}^{2}+\frac{1}{2 \lambda_{i}} \bar{\delta}_{i}^{2}$ 
where $\lambda_{i}$ is a positive design constant.

Based on (25) and (28), we get

$$
\begin{gathered}
\dot{V}_{i} \leq z_{i}\left[g_{i}\left(\underline{x}_{i}\right) x_{i+1}+W_{i}^{* T} S_{i}\left(Z_{i}\right)-\varrho_{i 1}-\varepsilon_{i}\right] \\
+\tilde{W}_{i}^{T} \Gamma_{i}^{-1} \dot{\hat{W}}_{i}+\frac{\lambda_{i}}{2} z_{i}^{2}+\frac{1}{2 \lambda_{i}} \bar{\delta}_{i}^{2} .
\end{gathered}
$$

Designing the virtual control law $\alpha_{i}$ and the relative neural weight updated law $\dot{\hat{W}}_{i}$ as

$\alpha_{i}=N\left(\varphi_{i}\right) \psi_{i}$

$\psi_{i}=k_{i} z_{i}+\hat{W}_{i}^{T} S_{i}\left(Z_{i}\right)-\varrho_{i 1}+\left(\frac{1}{2}+\frac{\lambda_{i}}{2}+\frac{\mu_{i}}{2}\right) z_{i}$

$\dot{\varphi}_{i}=z_{i} \psi_{i}$

$\dot{\hat{W}}_{i}=\Gamma_{i}\left[z_{i} S_{i}\left(Z_{i}\right)-\sigma_{i} \hat{W}_{i}\right]$

where $k_{i}, \mu_{i}$ and $\sigma_{i}$ are all positive design parameters.

According to (30)-(32), we can conclude that

$$
\begin{aligned}
z_{i} g_{i}\left(\underline{x}_{i}\right) x_{i+1}= & z_{i}\left[g_{i}\left(\underline{x}_{i}\right) z_{i+1}-\hat{W}_{i}^{T} S_{i}\left(Z_{i}\right)+\varrho_{i 1}\right] \\
& -\left(k_{i}+\frac{\lambda_{i}}{2}+\frac{\mu_{i}}{2}+\frac{1}{2}\right) z_{i}^{2} \\
& +\left(g_{i}\left(\underline{x}_{i}\right) N\left(\varphi_{i}\right)+1\right) \dot{\varphi}_{i} .
\end{aligned}
$$

Substituting (33) and (34) into (29), it can be obtained that

$$
\begin{array}{r}
\dot{V}_{i} \leq z_{i} g_{i}\left(\underline{x}_{i}\right) z_{i+1}+\left(g_{i}\left(\underline{x}_{i}\right) N\left(\varphi_{i}\right)+1\right) \dot{\varphi}_{i}-z_{i} \varepsilon_{i} \\
-\sigma_{i} \tilde{W}_{i}^{T} \hat{W}_{i}-\left(k_{i}+\frac{\mu_{i}}{2}+\frac{1}{2}\right) z_{i}^{2}+\frac{1}{2 \lambda_{i}} \bar{\delta}_{i}^{2} .
\end{array}
$$

With the help of Young's inequality, we have

$$
\begin{aligned}
& z_{i} g_{i}\left(\underline{x}_{i}\right) z_{i+1} \leq \frac{1}{2} g_{i}^{2}\left(\underline{x}_{i}\right) z_{i+1}^{2}+\frac{1}{2} z_{i}^{2} \\
& -z_{i} \varepsilon_{i} \leq \frac{\mu_{i}}{2} z_{i}^{2}+\frac{1}{2 \mu_{i}} \bar{\varepsilon}_{i}^{2} \\
& -\sigma_{i} \tilde{W}_{i}^{T} \hat{W}_{i} \leq-\frac{\sigma_{i}}{2}\left\|\tilde{W}_{i}\right\|^{2}+\frac{\sigma_{i}}{2}\left\|W_{i}^{*}\right\|^{2} .
\end{aligned}
$$

By substituting (36) into (35), one gets

$$
\begin{aligned}
\dot{V}_{i} \leq & -k_{i} z_{i}^{2}-\frac{\sigma_{i}}{2}\left\|\tilde{W}_{i}\right\|^{2}+\frac{\sigma_{i}}{2}\left\|W_{i}^{*}\right\|^{2}+\frac{1}{2 \mu_{i}} \bar{\varepsilon}_{i}^{2} \\
& +\frac{1}{2 \lambda_{i}} \bar{\delta}_{i}^{2}+\left(g_{i}\left(\underline{x}_{i}\right) N\left(\varphi_{i}\right)+1\right) \dot{\varphi}_{i}+\frac{1}{2} g_{i}^{2}\left(\underline{x}_{i}\right) z_{i+1}^{2} \\
\leq & -p_{i} V_{i}+q_{i}+\left(g_{i}\left(\underline{x}_{i}\right) N\left(\varphi_{i}\right)+1\right) \dot{\varphi}_{i}+\frac{1}{2} g_{i}^{2}\left(\underline{x}_{i}\right) z_{i+1}^{2}
\end{aligned}
$$

where $q_{i}=\sigma_{i}\left\|W_{i}^{*}\right\|^{2} / 2+\bar{\varepsilon}_{i}^{2} / 2 \mu_{i}+\bar{\delta}_{i}^{2} / 2 \lambda_{i}$ and $p_{i}=$ $\min \left\{2 k_{i}, \sigma_{i} / \lambda_{\max }\left(\Gamma_{i}^{-1}\right)\right\}$.

Step $n$ : For $z_{n}=x_{n}-\alpha_{n-1}$, its dynamics along (1) can be expressed as

$\dot{z}_{n}=f_{n}\left(\underline{x}_{n}\right)+g_{n}\left(\underline{x}_{n}\right) u-\dot{\alpha}_{n-1}$.
Similar to Step $i$, the FOSMD is employed to acquire $\dot{\alpha}_{n-1}$, which can avoid tedious computation:

$\left\{\begin{array}{l}\dot{\vartheta}_{n 1}=\varrho_{n 1} \\ \varrho_{n 1}=-\omega_{n 1}\left|\vartheta_{n 1}-\alpha_{n-1}\right|^{\frac{1}{2}} \operatorname{sign}\left(\vartheta_{n 1}-\alpha_{n-1}\right)+\vartheta_{n 2} \\ \dot{\vartheta}_{n 2}=-\omega_{n 2} \operatorname{sign}\left(\vartheta_{n 2}-\varrho_{n 1}\right)\end{array}\right.$

where $\vartheta_{n 1}, \vartheta_{n 2}$ and $\varrho_{n 1}$ denote the states of FOSMD (39), $\omega_{n 1}$ and $\omega_{n 2}$ are both positive design parameters.

Then, it can be concluded from (39) and Lemma 3-4 that

$\dot{\alpha}_{n-1}=\varrho_{n 1}+\varepsilon_{n}$

where $\varepsilon_{n}$ satisfies $\left|\varepsilon_{n}\right| \leq \bar{\varepsilon}_{n}$ with $\bar{\varepsilon}_{n}$ being a positive constant.

Let the Lyapunov function candidate as

$V_{n}=\frac{1}{2} z_{n}^{2}+\frac{1}{2} \tilde{W}_{n}^{T} \Gamma_{n}^{-1} \tilde{W}_{n}+\frac{1}{2 G} \tilde{g}_{n 2}^{2}$

where $\Gamma_{n}=\Gamma_{n}^{T}>0$ is a constant matrix, $G$ is a positive design constant, $\tilde{W}_{n}=\hat{W}_{n}-W_{n}^{*}$ stands for the weight estimation error with $\hat{W}_{n}$ being the estimation of $W_{n}^{*}$, $\tilde{g}_{n 2}=\hat{g}_{n 2}-g_{n 2}$ with $\hat{g}_{n 2}$ being the estimation of $g_{n 2}$.

Furthermore, based on (38), one can deduce

$$
\begin{aligned}
\dot{V}_{n}=z_{n} & {\left[g_{n}\left(\underline{x}_{n}\right) u+W_{n}^{* T} S_{n}\left(Z_{n}\right)+\delta_{n}\left(Z_{n}\right)\right.} \\
& \left.-\dot{\alpha}_{n-1}\right]+\tilde{W}_{n}^{T} \Gamma_{n}^{-1} \dot{\hat{W}}_{n}+G^{-1} \tilde{g}_{n 2} \dot{\hat{g}}_{n 2}
\end{aligned}
$$

where $W_{n}^{* T} S_{n}\left(Z_{n}\right)$ is adopted to approximate $f_{n}\left(\underline{x}_{n}\right)$ with $Z_{n}=\left[x_{1}, x_{2}, \ldots, x_{n}\right]^{T} \in \mathbb{R}^{n}$ and $\left|\delta_{n}\left(Z_{n}\right)\right| \leq \bar{\delta}_{n}$.

Based on Young's inequality, we get

$z_{n} \delta_{n}\left(Z_{n}\right) \leq \frac{\lambda_{n}}{2} z_{n}^{2}+\frac{1}{2 \lambda_{n}} \bar{\delta}_{n}^{2}$

where $\lambda_{n}>0$ is a design parameter.

By substituting (40) and (43) into (42), we have

$$
\begin{aligned}
\dot{V}_{n} \leq & z_{n}\left[g_{n}\left(\underline{x}_{n}\right) u+W_{n}^{* T} S_{n}\left(Z_{n}\right)-\varrho_{n 1}-\varepsilon_{n}\right] \\
& +\frac{\lambda_{n}}{2} z_{n}^{2}+\tilde{W}_{n}^{T} \Gamma_{n}^{-1} \dot{\hat{W}}_{n}+G^{-1} \tilde{g}_{n 2} \dot{\hat{g}}_{n 2} \\
& +\frac{1}{2 \lambda_{n}} \bar{\delta}_{n}^{2} .
\end{aligned}
$$

The adaptive controller $\nu(t)$ are chosen as

$$
\begin{aligned}
\nu(t)= & N\left(\varphi_{n}\right) \psi_{n} \\
\psi_{n}= & k_{n} z_{n}+\hat{W}_{n}^{T} S_{n}\left(Z_{n}\right)-\varrho_{n 1} \\
& +\hat{g}_{n 2} \bar{\theta} \tanh \left(\frac{\bar{\theta} z_{n}}{\pi}\right)+\left(\frac{\lambda_{n}}{2}+\frac{\mu_{n}}{2}\right) z_{n} \\
\dot{\varphi}_{n}= & z_{n} \psi_{n}
\end{aligned}
$$


with

$$
\left\{\begin{array}{l}
\dot{\hat{W}}_{n}=\Gamma_{n}\left[z_{n} S_{n}\left(Z_{n}\right)-\sigma_{n} \hat{W}_{n}\right] \\
\dot{\hat{g}}_{n 2}=G\left[\bar{\theta} z_{n} \tanh \left(\frac{\bar{\theta} z_{n}}{\pi}\right)-\gamma \hat{g}_{n 2}\right]
\end{array}\right.
$$

where $k_{n}, \sigma_{n}, \pi$ and $\gamma$ are all positive design parameters, $\bar{\theta}$ will be given latter.

Constructing the following ET mechanism

$$
\begin{aligned}
u(t) & =\nu\left(t_{k}\right), \forall t \in\left[t_{k}, t_{k+1}\right) \\
t_{k+1} & =\inf \{t \in \mathbb{R}|| \epsilon(t)|\geq \rho| u(t) \mid+\theta\}
\end{aligned}
$$

where $\epsilon(t)=\nu(t)-u(t)$ represents the measurement error, $t_{k}, k \in \mathbb{Z}^{+}$stands for the triggering instant, $0<$ $\rho<1, \theta>0$ and $\bar{\theta}>\theta /(1-\rho)$ are all design constants.

\section{4 main results}

Theorem 1 For the SFNSs (1) under Assumptions 12 with prescribed tracking performance (3) and unknown control direction. If we construct the virtual laws (15) and (30), the adaptive controller (45) with the eventtriggering rules given in (49)-(50), the adaptive laws (18), (33) and (48), then for any initial values $e_{1}(0)$ satisfying (3), the presented ETC scheme can guarantee:

1) all the closed-loop signals are bounded on $[0,+\infty)$, and the tracking error $e_{1}$ converges to a small residual set of zero with performance constraint (3).

2) For $\forall k \in \mathbb{Z}^{+}$, there exists a minimum inter-execution intervals $t^{*}>0$ such that $\left\{t_{k+1}-t_{k}\right\} \geq t^{*}$, i.e., the Zeno phenomenon does not happen.

Proof: It can be concluded from (50) that $\nu(t)=$ $\left(1+\zeta_{1}(t) \rho\right) u(t)+\zeta_{2}(t) \theta, \forall t \in\left[t_{k}, t_{k+1}\right)$, where $\left|\zeta_{1}(t)\right| \leq 1$ and $\left|\zeta_{2}(t)\right| \leq 1$ stand for the time-varying parameters. Then, we get

$u(t)=\frac{\nu(t)-\zeta_{2}(t) \theta}{1+\zeta_{1}(t) \rho}$.

Based on (45)-(47) and (51), one has

$$
\begin{aligned}
z_{n} g_{n}\left(\underline{x}_{n}\right) u= & z_{n}\left[-\frac{g_{n}\left(\underline{x}_{n}\right) \zeta_{2}(t) \theta}{1+\zeta_{1}(t) \rho}-\hat{W}_{n}^{T} S_{n}\left(Z_{n}\right)\right. \\
& \left.+\varrho_{n 1}-\hat{g}_{n 2} \bar{\theta} \tanh \left(\frac{\bar{\theta} z_{n}}{\pi}\right)\right] \\
& +\left[\frac{g_{n}\left(\underline{x}_{n}\right)}{1+\zeta_{1}(t) \rho} N\left(\varphi_{n}\right)+1\right] \dot{\varphi}_{n} \\
& -\left(k_{n}+\frac{\lambda_{n}}{2}+\frac{\mu_{n}}{2}\right) z_{n}^{2} .
\end{aligned}
$$

Substituting (48) and (52) into (44), one can obtain

$$
\begin{aligned}
\dot{V}_{n} \leq & \left(\frac{g_{n}\left(\underline{x}_{n}\right)}{1+\zeta_{1}(t) \rho} N\left(\varphi_{n}\right)+1\right) \dot{\varphi}_{n}-\left(k_{n}+\frac{\mu_{n}}{2}\right) z_{n}^{2} \\
& +z_{n}\left[-\frac{g_{n}\left(\underline{x}_{n}\right) \zeta_{2}(t) \theta}{1+\zeta_{1}(t) \rho}-g_{n 2} \bar{\theta} \tanh \left(\frac{\bar{\theta} z_{n}}{\pi}\right)\right] \\
& -z_{n} \varepsilon_{n}-\sigma_{n} \tilde{W}_{n}^{T} \hat{W}_{n}-\gamma \tilde{g}_{n 2} \hat{g}_{n 2}+\frac{1}{2 \lambda_{n}} \bar{\delta}_{n}^{2}
\end{aligned}
$$

Since $\left|\zeta_{1}(t)\right| \leq 1,\left|\zeta_{2}(t)\right| \leq 1,0<\rho<1$, then according to Lemma 5 , we have

$z_{n}\left[-\frac{g_{n}\left(\underline{x}_{n}\right) \zeta_{2}(t) \theta}{1+\zeta_{1}(t) \rho}-g_{n 2} \bar{\theta} \tanh \left(\frac{\bar{\theta} z_{n}}{\pi}\right)\right] \leq g_{n 2} \pi^{*}$

where $\pi^{*}=0.2785 \pi$.

With the aid of Young's inequality, we have

$$
\begin{aligned}
-z_{n} \varepsilon_{n} & \leq \frac{\mu_{n}}{2} z_{n}^{2}+\frac{1}{2 \mu_{n}} \bar{\varepsilon}_{n}^{2} \\
-\sigma_{n} \tilde{W}_{n}^{T} \hat{W}_{n} & \leq-\frac{\sigma_{n}}{2}\left\|\tilde{W}_{n}\right\|^{2}+\frac{\sigma_{n}}{2}\left\|W_{n}^{*}\right\|^{2} \\
-\gamma \tilde{g}_{n 2} \hat{g}_{n 2} & \leq-\frac{\gamma}{2} \tilde{g}_{n 2}^{2}+\frac{\gamma}{2} g_{n 2}^{2} .
\end{aligned}
$$

Substituting (54) and (55) into (53), one has

$$
\begin{aligned}
\dot{V}_{n} \leq & -k_{n} z_{n}^{2}-\frac{\sigma_{n}}{2}\left\|\tilde{W}_{n}\right\|^{2}-\frac{\gamma}{2} \tilde{g}_{n 2}^{2}+\frac{\sigma_{n}}{2}\left\|W_{n}^{*}\right\|^{2} \\
& +\frac{\gamma}{2} g_{n 2}^{2}+\frac{1}{2 \lambda_{n}} \bar{\delta}_{n}^{2}+\frac{1}{2 \mu_{n}} \bar{\varepsilon}_{n}^{2}+g_{n 2} \pi^{*} \\
& +\left(\frac{g_{n}\left(\underline{x}_{n}\right)}{1+\zeta_{1}(t) \rho} N\left(\varphi_{n}\right)+1\right) \dot{\varphi}_{n} \\
\leq & -p_{n} V_{n}+q_{n}+\left(b\left(\underline{x}_{n}\right) N\left(\varphi_{n}\right)+1\right) \dot{\varphi}_{n}
\end{aligned}
$$

where $q_{n}=\sigma_{n}\left\|W_{n}^{*}\right\|^{2} / 2+\gamma g_{n 2}^{2} / 2+\bar{\delta}_{n}^{2} / 2 \lambda_{n}+\bar{\varepsilon}_{n}^{2} / 2 \mu_{n}+$ $g_{n 2} \pi^{*}, p_{n}=\min \left\{2 k_{n}, \sigma_{n} / \lambda_{\max }\left(\Gamma_{n}^{-1}\right), \gamma G\right\}$ and $b\left(\underline{x}_{n}\right)=$ $g_{n}\left(\underline{x}_{n}\right) /\left(1+\zeta_{1}(t) \rho\right)$.

Since $0<g_{n 1} \leq\left|g_{n}\left(\underline{x}_{n}\right)\right| \leq g_{n 2},\left|\zeta_{1}(t)\right| \leq 1$ and $0<\rho<1$, we can conclude that $b\left(\underline{x}_{n}\right)$ is bounded and there exists unknown positive constants $\underline{b}$ and $\bar{b}$ such that $\underline{b} \leq\left|b\left(\underline{x}_{n}\right)\right| \leq \bar{b}$. Moreover, the sign of $b\left(\underline{x}_{n}\right)$ is as same as $g_{n}\left(\underline{x}_{n}\right)$.

Multiplying both sides of (56) by $e^{p_{n} t}$ and integrating it over the interval $[0, t]$, it follows that

$$
\begin{aligned}
V_{n}(t) \leq & \frac{q_{n}}{p_{n}}+\left(V_{n}(0)-\frac{q_{n}}{p_{n}}\right) e^{-p_{n} t} \\
& +e^{-p_{n} t} \int_{0}^{t}\left(b\left(\underline{x}_{n}\right) N\left(\varphi_{n}\right)+1\right) \dot{\varphi}_{n} e^{p_{n} \tau} d \tau \\
\leq & \frac{q_{n}}{p_{n}}+V_{n}(0) \\
& +e^{-p_{n} t} \int_{0}^{t}\left(b\left(\underline{x}_{n}\right) N\left(\varphi_{n}\right)+1\right) \dot{\varphi}_{n} e^{p_{n} \tau} d \tau .
\end{aligned}
$$


Then, it can be concluded from (57) and Lemma 2 that $V_{n}(t)$ and $\varphi_{n}(t)$ are bounded on $\left[0, t_{f}\right)$. Consequently, $z_{n}, \hat{W}_{n}$ and $\hat{g}_{n 2}$ are also bounded on $\left[0, t_{f}\right)$.

Utilizing the similar way, we can obtain from (37) that

$$
\begin{aligned}
V_{i}(t) \leq & \frac{q_{i}}{p_{i}}+\left(V_{i}(0)-\frac{q_{i}}{p_{i}}\right) e^{-p_{i} t} \\
& +e^{-p_{i} t} \int_{0}^{t}\left(g_{i}\left(\underline{x}_{i}\right) N\left(\varphi_{i}\right)+1\right) \dot{\varphi}_{i} e^{p_{i} \tau} d \tau \\
& +\frac{1}{2} e^{-p_{i} t} \int_{0}^{t} g_{i}^{2}\left(\underline{x}_{i}\right) z_{i+1}^{2} e^{p_{i} \tau} d \tau
\end{aligned}
$$

Let $i=n-1$ in (58), we have

$$
\begin{aligned}
V_{n-1}(t) \leq & \frac{q_{n-1}}{p_{n-1}}+V_{n-1}(0)+e^{-p_{n-1} t} \\
& \times \int_{0}^{t}\left(g_{n-1}\left(\underline{x}_{n-1}\right) N\left(\varphi_{n-1}\right)+1\right) \dot{\varphi}_{n-1} e^{p_{n-1} \tau} d \tau \\
& +\frac{1}{2} e^{-p_{n-1} t} \int_{0}^{t} g_{n-1}^{2}\left(\underline{x}_{n-1}\right) z_{n}^{2} e^{p_{n-1} \tau} d \tau
\end{aligned}
$$

Furthermore, from Assumption 1, one has

$$
\begin{aligned}
& \frac{1}{2} e^{-p_{n-1} t} \int_{0}^{t} g_{n-1}^{2}\left(\underline{x}_{n-1}\right) z_{n}^{2} e^{p_{n-1} \tau} d \tau \\
& \leq \frac{1}{2} e^{-p_{n-1} t} g_{(n-1) 2}^{2} \sup _{\tau \in[0, t]} z_{n}^{2}(\tau) \int_{0}^{t} e^{p_{n-1} \tau} d \tau \\
& \leq \frac{g_{(n-1) 2}^{2}}{2 p_{n-1}} \sup _{\tau \in[0, t]} z_{n}^{2}(\tau) .
\end{aligned}
$$

Based on the boundedness of $z_{n}$ and (60), we can deduce that $e^{-p_{n-1} t} \int_{0}^{t} g_{n-1}^{2}\left(\underline{x}_{n-1}\right) z_{n}^{2} e^{p_{n-1} \tau} d \tau / 2$ is bounded on $\left[0, t_{f}\right)$. Then, we can get from (59) and Lemma 2 that $V_{n-1}(t)$ and $\varphi_{n-1}(t)$ are bounded on $\left[0, t_{f}\right)$. As a consequence, $z_{n-1}$ and $\hat{W}_{n-1}$ are bounded on $\left[0, t_{f}\right)$. Similarly, we can prove that $\varphi_{i}(t)$ and $V_{i}(t)$ are bounded on $\left[0, t_{f}\right)$, and therefore, $z_{i}$ and $\hat{W}_{i}$ with $i=1,2, \ldots, n-$ 2 are also bounded on $\left[0, t_{f}\right)$. According to [44], we can get $t_{f}=+\infty$. Moreover, based on the error transformation (5), it is clear that $e_{1}$ is bounded due to the boundedness of $z_{1}$ and $\phi(t)$. By combining the boundedness of $y_{d}$ and $e_{1}=x_{1}-y_{d}$, it is easy to obtain that $x_{1}$ is bounded. Owing to the boundedness of $x_{1}, \hat{W}_{1}, \varphi_{1}, y_{d}, \dot{y}_{d}, \phi(t)$ and $\dot{\phi}(t)$, it follows from (15) that virtual controller $\alpha_{1}$ is boundeded on $[0,+\infty)$. Then, according to Lemma $3-4$ and (24), we can conclude that $\vartheta_{21}$ is also bounded on $[0,+\infty)$. Furthermore, since $z_{2}=x_{2}-\alpha_{1}$, it is easy to infer that $x_{2}$ is bounded on $[0,+\infty)$. Noting that $\dot{\alpha}_{1}$ is a continuous function of the bounded signals $x_{1}, x_{2}$, $\hat{W}_{1}, \varphi_{1}, y_{d}, \dot{y}_{d}, \ddot{y}_{d}, \phi(t), \dot{\phi}(t)$ and $\ddot{\phi}(t)$, thus we can get $\dot{\alpha}_{1}$ is bounded on $[0,+\infty)$. Then, by $(25)$, it is obvious that $\rho_{21}$ is bounded on $[0,+\infty)$. Moreover, we can concluded that $\vartheta_{22}$ is also bounded on $[0,+\infty)$. Similarly, we can progressively demonstrate that $x_{i}, \vartheta_{i 1}, \vartheta_{i 2}, \varrho_{i 1}$, $\alpha_{j}$ and $u$ with $i=3,4, \ldots, n, j=2,3, \ldots, n-1$ are also bounded on $[0,+\infty)$. Let $q_{1}^{*}=q_{1} / p_{1}$, then by $(58)$ and the definition of $V_{1}$ given in (9), we can obtain that when $t \rightarrow \infty$

$\left|z_{1}\right| \leq \sqrt{2 q_{1}^{*}}$

By choosing suitable design parameters, it follows from (61) that $z_{1}$ can converge to a small residual set of zero. Then, according to the error transformation (5), we can easily conclude that tracking error $e_{1}$ also converges to a small residual set of zero. Therefore, all the closed-loop signals are bounded on $[0,+\infty)$, and $e_{1}$ can converge to a small residual set of zero with the prescribed performance $(3)$.

Since $\epsilon(t)=\nu(t)-u(t), \forall t \in\left[t_{k}, t_{k+1}\right)$, and then we have

$\frac{d|\epsilon|}{d t}=\frac{d}{d t}(\epsilon \times \epsilon)^{\frac{1}{2}}=\operatorname{sign}(\epsilon) \dot{\epsilon} \leq|\dot{\nu}|$.

By (45), one obtains

$\dot{\nu}=\frac{d N\left(\varphi_{n}\right)}{d \varphi_{n}} \dot{\varphi}_{n} \psi_{n}+N\left(\varphi_{n}\right) \dot{\psi}_{n}$

Based on the boundedness of $y_{d}, \dot{y}_{d}, \ddot{y}_{d}, \phi(t), \dot{\phi}(t), \ddot{\phi}(t)$, $\hat{g}_{n 2}, x_{i}, \hat{W}_{i}, \varphi_{i}, \varrho_{j 1}, \vartheta_{j 1}$ and $\vartheta_{j 2}, i=1,2, \ldots, n, j=$ $2,3, \ldots, n$, it is obvious that the continues function $\dot{\nu}$ is bounded. Thus, there exists a constant $\varpi>0$ such that $|\dot{\nu}| \leq \varpi$. Noting that $\epsilon\left(t_{k}\right)=0$ and $\lim _{t \rightarrow t_{k+1}} \epsilon(t)=$ $\rho|u(t)|+\theta>\theta$, the minimum inter-execution intervals $t^{*} \geq \theta / \varpi$ can be obtained. As a consequence, the Zeno phenomenon does not happen.

Remark 2 From the ET controller (45)-(50), it is obvious that the control signal is sent to the actuator in an aperiodic way, which significantly economizes the communication resource between the controller and actuator. However, due to the unknown direction of control gain $g_{n}\left(\underline{x}_{n}\right)$, it is quite difficult to construct a suitable controller to compensate the measurement error caused by the ET condition (50). To deal with such a difficulty, two effects have been taken in this paper: 1) the nonlinear term $-g_{n 2} \bar{\theta} \tanh \left(\frac{\bar{\theta} z_{n}}{\pi}\right)$ is introduced into (53), which effectively compensates the measurement error based on Lemma 5, see (54) for details; 2) an ingenious adaptive law $\dot{\hat{g}}_{n 2}$ is designed in (48) to estimate the unknown upper bound $g_{n 2}$ of $g_{n}\left(\underline{x}_{n}\right)$, which makes it possible to co-design the controller and the ET mechanism. As a consequence, the effect of the measurement error on the system stability is handled effectively. 


\section{Simulation studies}

For demonstrating the effectiveness of our presented method, the following two simulation examples are considered in this section.

\subsection{Numerical example}

Consider the SFNSs [45] as follows

$$
\left\{\begin{array}{l}
\dot{x}_{1}=\sin \left(x_{1}\right)+\left(x_{1}^{2}+1\right) x_{2} \\
\dot{x}_{2}=0.2 x_{1} x_{2}+\left(\cos \left(x_{1} x_{2}\right)+3\right) u \\
y=x_{1}
\end{array}\right.
$$

The main goal of this simulation study is to make the system output $y$ track the reference signal $y_{d}=0.8 \sin (t)$ while reduce the communication burden. Meanwhile, the tracking error $e_{1}$ satisfies the predefined performance constraint $-\varsigma_{1} \phi(t)<e_{1}(t)<\varsigma_{2} \phi(t)$, where $\varsigma_{1}=$ $3, \varsigma_{2}=4$, and $\phi(t)$ is defined in $(2)$ with $\phi_{1}=0.8$, $\phi_{2}=0.05$ and $T_{c}=5$. The initial values are $x_{1}(0)=$ $-0.4, x_{2}(0)=0, \vartheta_{21}(0)=1, \vartheta_{22}(0)=2, \varphi_{1}(0)=1.6$, $\varphi_{2}(0)=-4.2, \hat{g}_{22}(0)=2, \hat{W}_{1}(0)=0$ and $\hat{W}_{2}(0)=0$. The design constants are selected as $k_{1}=k_{2}=2$, $\lambda_{1}=\lambda_{2}=1, \mu_{2}=0.1, \sigma_{1}=\sigma_{2}=0.00001, G=5$, $\gamma=0.00001, \pi=40, \rho=0.11, \theta=1.9$, and $\bar{\theta}=2.6$. Moreover, we construct the RBF NN $\hat{W}_{1}^{T} S\left(Z_{1}\right)$ utilizing 81 nodes with centers $\Theta_{1}$ distributed on $[-1,1] \times$ $[-1,1] \times[-1,1] \times[0,1]$ and width $\Delta_{1}=[1,1,1,0.5]^{T}$ and the $\hat{W}_{2}^{T} S\left(Z_{2}\right)$ utilizing 15 nodes with centers $\Theta_{2}$ distributed on $[-1,1] \times[-1.2,2.5]$ and width $\Delta_{2}=[1,1]^{T}$.

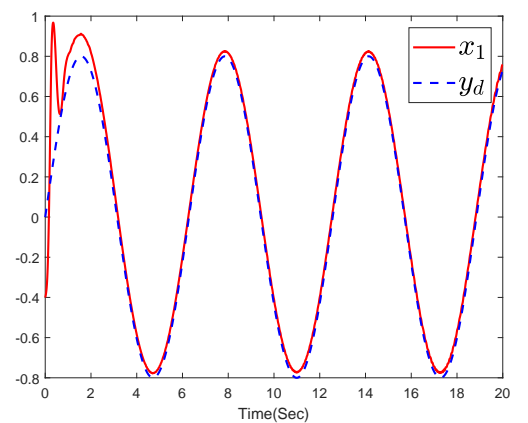

Fig. 1 Curves of $y=x_{1}$ and $y_{d}$.

Simulation results are shown in Figs. 1-5. From Figs. 12 , we can see that system output $y$ can efficaciously track $y_{d}$ and the tracking error $e_{1}$ satisfies the predefined performance constraint. Fig. 3 illustrates the curve of the ETC signal $u(t)$. It can be concluded from Fig. 3 that the communication burden between the controller and actuator are significantly reduced. Fig. 4 displays the inter-event times, which indicates the Zeno

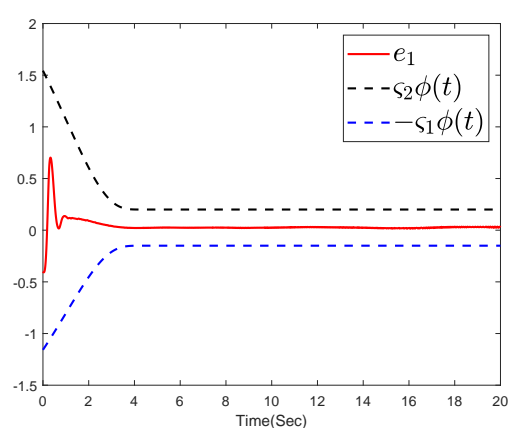

Fig. 2 Curves of $e_{1}$ with predefined performance constraint.

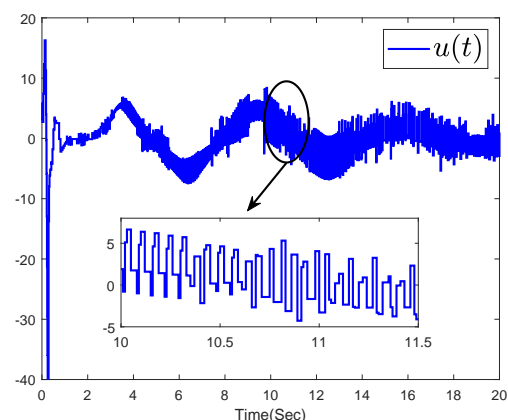

Fig. 3 Curve of control signal $u(t)$.

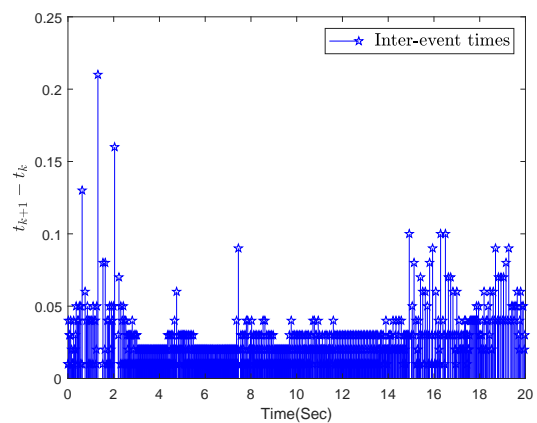

Fig. 4 Inter-event times.

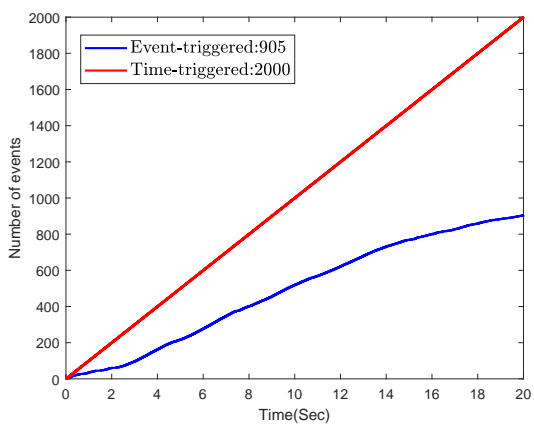

Fig. 5 Cumulative number of events.

phenomenon doesn't happen. The cumulative number of ET instants is shown in Fig. 5. In comparison with the time-triggered control scheme, the ETC scheme p- 
resented in this paper only requires 905 times signal transmission, which economizes nearly $55 \%$ communication resource.

5.2 The Spring-mass-damper mechanical vibration system

To further show the applicability of our presented ETC approach, we consider the spring-mass-damper mechanical vibration system [26] as follows

$$
\left\{\begin{array}{l}
\dot{x}_{1}=x_{2} \\
\dot{x}_{2}=-\frac{J_{s}}{M} x_{1}-\frac{C_{d}}{M} x_{2}+\frac{1}{M} u \\
y=x_{1}
\end{array}\right.
$$

where $x_{1}$ and $x_{2}$, denote, respectively, the position and velocity. The physical parameters are selected as $M=$ $1, C_{d}=2$ and $J_{s}=8$, which are as same as these in [26]. The desired reference output is $y_{d}=1.6 \sin (t)+$ $1.6 \sin (0.5 t)$.

In the simulation, the predefined performance constraint is given as $-\varsigma_{1} \phi(t)<e_{1}(t)<\varsigma_{2} \phi(t)$ with $\varsigma_{1}=$ $3.2, \varsigma_{2}=5$, and the design constants of $\phi(t)$ are $\phi_{1}=$ $0.75, \phi_{2}=0.045$ and $T_{c}=3.5$. The initial values are $x_{1}(0)=0.8, x_{2}(0)=0, \vartheta_{21}(0)=0, \vartheta_{22}(0)=2, \varphi_{1}(0)=$ $1.5, \varphi_{2}(0)=3, \hat{g}_{22}(0)=0$ and $\hat{W}_{2}(0)=0$. Moreover, we construct the $\mathrm{RBF} \mathrm{NN} \hat{W}_{2}^{T} S\left(Z_{2}\right)$ utilizing $42 \mathrm{n}-$ odes with centers $\Theta_{2}$ distributed on $[-3,3] \times[-2,3.5]$ and width $\Delta_{2}=[1.25,1.25]^{T}$. Then, by selecting the suitable parameters $k_{1}=2, k_{2}=5.5, \lambda_{2}=\mu_{2}=1$, $\sigma_{2}=0.00001, G=3, \gamma=0.00001, \pi=10, \rho=0.1$, $\theta=2$, and $\bar{\theta}=4$, we can obtain the good simulation results, which are shown in Figs. 6-10. It can be concluded from Figs. 6-10 that the presented control scheme ensures the predefined performance constraint and economizes nearly $61 \%$ communication resource.

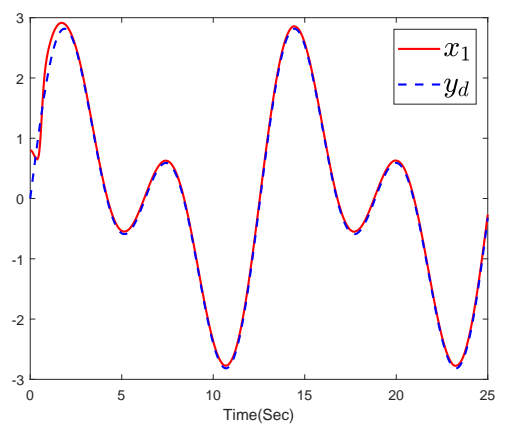

Fig. 6 Curves of $y=x_{1}$ and $y_{d}$.

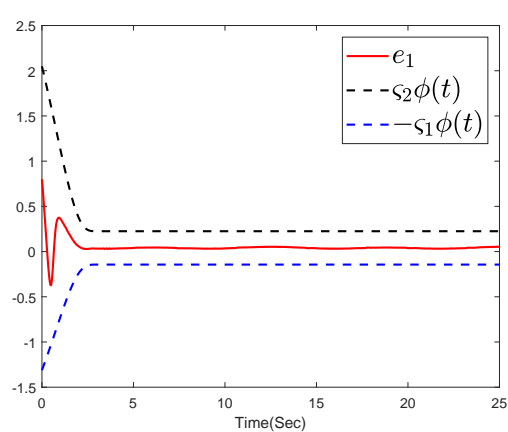

Fig. 7 Curves of $e_{1}$ with predefined performance constraint.

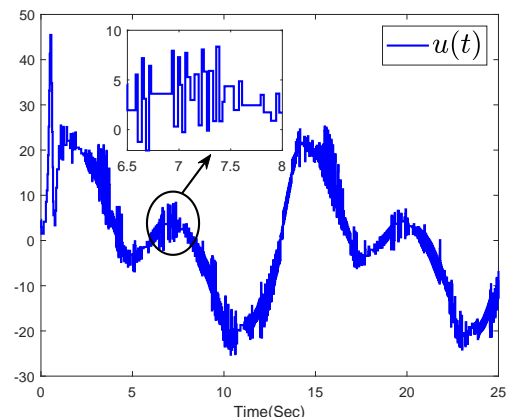

Fig. 8 Curve of control signal $u(t)$.

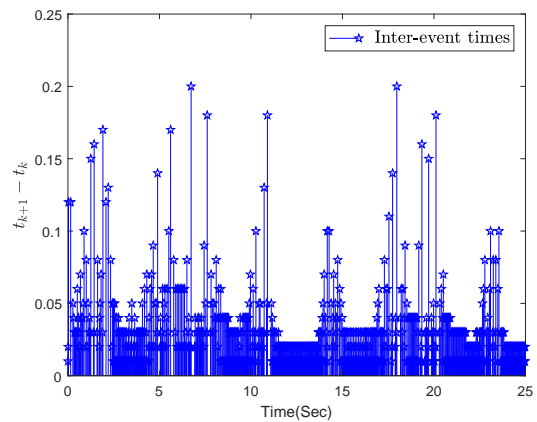

Fig. 9 Inter-event times.

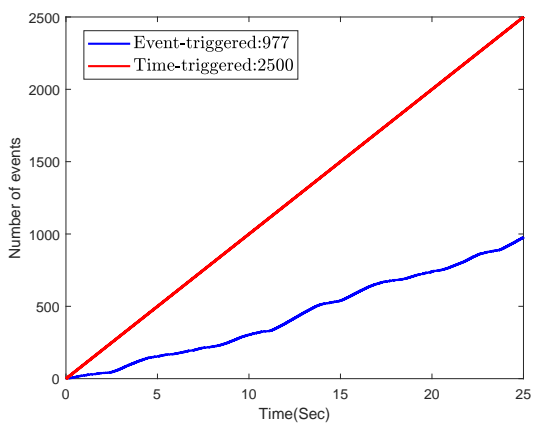

Fig. 10 Cumulative number of events.

\section{Conclusions}

This paper considered the finite-time performance guaranteed ETC problem for SFNSs with unknown con- 
trol direction. An easy-to-implement finite-time performance function has been constructed to depict the predefined performance constraint, and then a related lemma has been developed to guarantee the stability of the considered closed-loop error system. Based on the error transformation technique, the original constrained tracking error has been transformed into an equivalent unconstrained one. Moreover, the introduction of the FOSMD avoided the issue of "explosion of complexity", thereby making the controller design and stability analysis easier. Meanwhile, an ingenious adaptive law has been developed to estimate the upper bound of the actual control coefficient. Subsequently, a novel performance guaranteed ETC scheme has been proposed by the combination of the adaptive law, which has achieved the predefined tracking performance, compensated the measurement error and economized the communication resource.

\section{Data Availability Statement}

Data sharing is not applicable to this paper because no datasets are generated or analyzed during the current study.

\section{Conflicts of interest}

The authors declare that they have no conflict of interest.

\section{Appendices}

\section{Proof of Lemma 1}

Firstly, we demonstrate that $\phi(t)$ is continuously differentiable and bounded for all $t \geq 0$. From (2), we know that $\phi(t)$ is continuously differentiable on the intervals $\left[0, T_{c}\right)$ and $\left[T_{c},+\infty\right)$. Thus, the key is to demonstrate that $\phi(t)$ is continuously differentiable at time $T_{c}$. By combining the definition of derivation and (2), one obtains

$$
\lim _{t \rightarrow T_{c}^{-}} \frac{\phi(t)-\phi\left(T_{c}\right)}{t-T_{c}}=\lim _{t \rightarrow T_{c}^{-}} \frac{-\tanh \left(\phi_{1}+\frac{t}{T_{c}-t}\right)+1}{t-T_{c}}
$$

Define $m=\phi_{1}+t /\left(T_{c}-t\right)$, then we can infer that $t \rightarrow$ $T_{c}^{-}$is equivalent to $m \rightarrow+\infty$, (62) can be expressed as

$$
\begin{aligned}
\lim _{t \rightarrow T_{c}^{-}} \frac{\phi(t)-\phi\left(T_{c}\right)}{t-T_{c}} & =\lim _{m \rightarrow+\infty} \frac{(1-\tanh (m))\left(1-\phi_{1}+m\right)}{-T_{c}} \\
& =\lim _{m \rightarrow+\infty} \frac{2\left(1-\phi_{1}+m\right)}{-T_{c}\left(e^{2 m}+1\right)} \\
& =0
\end{aligned}
$$

Based on (2), we can get $\lim _{t \rightarrow T_{c}^{+}}\left(\phi(t)-\phi\left(T_{c}\right)\right) /(t-$ $\left.T_{c}\right)=0$, and then it can be concluded that $\lim _{t \rightarrow T_{c}^{-}}(\phi(t)-$ $\left.\phi\left(T_{c}\right)\right) /\left(t-T_{c}\right)=\lim _{t \rightarrow T_{c}^{+}}\left(\phi(t)-\phi\left(T_{c}\right)\right) /\left(t-T_{c}\right)$. As a consequence, $\phi(t)$ is continuously differentiable at time $T_{c}$, which also means that $\phi(t)$ is continuously differentiable for all $t \geq 0$. Moreover, $\dot{\phi}(t)$ can be expressed as follows

$\dot{\phi}(t)=\left\{\begin{array}{cc}\frac{-T_{c}}{\left(T_{c}-t\right)^{2}}\left[1-\tanh ^{2}\left(\phi_{1}+\frac{t}{T_{c}-t}\right)\right], & 0 \leq t<T_{c} \\ 0 & , t \geq T_{c}\end{array}\right.$

From (64), we can obtain that $\dot{\phi}(t)<0$ on $\left[0, T_{c}\right)$ and $\dot{\phi}(t)=0$ on $\left[T_{c}, \infty\right)$. Then, it is easy to know that $\phi\left(T_{c}\right) \leq \phi(t) \leq \phi(0)$, which indicates that $\phi(t)$ is bounded for all $t \geq 0$.

Secondly, we demonstrate that $\dot{\phi}(t)$ is continuously differentiable and bounded for all $t \geq 0$. By (64), it is obvious that $\dot{\phi}(t)$ is continuously differentiable on $\left[0, T_{c}\right)$ and $\left[T_{c},+\infty\right)$. Thus, we only need to verify that $\dot{\phi}(t)$ is continuously differentiable at time $T_{c}$. By combining the definition of derivation with (64) and let $m=\phi_{1}+$ $t /\left(T_{c}-t\right)$, it follows that

$$
\begin{aligned}
\lim _{t \rightarrow T_{c}^{-}} \frac{\dot{\phi}(t)-\dot{\phi}\left(T_{c}\right)}{t-T_{c}} & =\lim _{m \rightarrow+\infty} \frac{\left(1-\tanh ^{2}(m)\right)\left(1-\phi_{1}+m\right)^{3}}{T_{c}^{2}} \\
& =\lim _{m \rightarrow+\infty} \frac{4\left(1-\phi_{1}+m\right)^{3}}{T_{c}^{2}\left(e^{2 m}+e^{-2 m}+2\right)} \\
& =0
\end{aligned}
$$

Since $\lim _{t \rightarrow T_{c}^{+}}\left(\dot{\phi}(t)-\dot{\phi}\left(T_{c}\right)\right) /\left(t-T_{c}\right)=0$, we can get that $\lim _{t \rightarrow T_{c}^{-}}\left(\dot{\phi}(t)-\dot{\phi}\left(T_{c}\right)\right) /\left(t-T_{c}\right)=\lim _{t \rightarrow T_{c}^{+}}(\dot{\phi}(t)-$ $\left.\dot{\phi}\left(T_{c}\right)\right) /\left(t-T_{c}\right)$. Apparently, $\dot{\phi}(t)$ is continuously differentiable at time $T_{c}$. Thus, $\dot{\phi}(t)$ is continuously differentiable for all $t \geq 0$ and $\ddot{\phi}(t)$ can be expressed as

$\ddot{\phi}(t)=\left\{\begin{array}{l}\frac{2 T_{c}}{\left(T_{c}-t\right)^{3}}\left[1-\tanh ^{2}\left(\phi_{1}+\frac{t}{T_{c}-t}\right)\right]\left[\frac{T_{c}}{T_{c}-t}\right. \\ \left.\times \tanh \left(\phi_{1}+\frac{t}{T_{c}-t}\right)-1\right], 0 \leq t<T_{c} \\ 0, \quad t \geq T_{c}\end{array}\right.$

Noting that $\dot{\phi}(t)$ is continuous for all $t \geq 0$, we can obtain that $\dot{\phi}(t)$ is bounded on $\left[0, T_{c}\right]$. Then, since $\dot{\phi}(t)=0$ holds for $t>T_{c}$, which further imply that $\dot{\phi}(t)$ is bounded for all $t \geq 0$.

Finally, we prove that $\ddot{\phi}(t)$ is continuously and bounded for all $t \geq 0$. On the basis of (66), we can see that 
$\ddot{\phi}(t)$ is continuous on $\left[0, T_{c}\right)$ and $\left[T_{c},+\infty\right)$. Consequently, the main task is to verify that $\ddot{\phi}(t)$ is continuous at time $T_{c}$.

Let $m=\phi_{1}+t /\left(T_{c}-t\right)$, then based on the definition of continuous function and (66), we have

$$
\begin{aligned}
& \lim _{t \rightarrow T_{c}^{-}} \ddot{\phi}(t) \\
= & \lim _{m \rightarrow+\infty} \frac{2\left(1-\phi_{1}+m\right)^{3}}{T_{c}^{2}}\left[1-\tanh ^{2}(m)\right] \\
& \times\left[\left(1-\phi_{1}+m\right) \tanh (m)-1\right] \\
= & \lim _{m \rightarrow+\infty} \frac{8\left(1-\phi_{1}+m\right)^{3}\left[\left(m-\phi_{1}\right)\left(e^{m}-e^{-m}\right)-2 e^{-m}\right]}{T_{c}^{2}\left(e^{2 m}+e^{-2 m}+2\right)\left(e^{m}+e^{-m}\right)} \\
= & 0
\end{aligned}
$$

Furthermore, it follows from (66) that $\lim _{t \rightarrow T_{c}^{+}} \ddot{\phi}(t)=$ 0 . Then, it can be seen that $\lim _{t \rightarrow T_{c}^{+}} \ddot{\phi}(t)=\lim _{t \rightarrow T_{c}^{-}} \ddot{\phi}(t)$, which means that $\ddot{\phi}(t)$ is continuous at time $T_{c}$. As such, $\ddot{\phi}(t)$ is continuous for all $t \geq 0$. Subsequently, it can be concluded that $\ddot{\phi}(t)$ is bounded on $\left[0, T_{c}\right]$. Besides, noting that $\ddot{\phi}(t)=0$ holds on $\left(T_{c}, \infty\right)$, it is obvious that $\ddot{\phi}(t)$ is bounded for all $t \geq 0$.

\section{References}

1. Liu, X., Jutan, A., Rohani, S.: Almost disturbance decoupling of MIMO nonlinear systems and application to chemical processes. Automatica. 40(3), 465-471 (2004)

2. Ling, S., Wang, H., Liu, P.X.: Adaptive fuzzy tracking control of flexible-joint robots based on command filtering. IEEE Transactions on Industrial Electronics. 67(5), 4046-4055 (2020)

3. Yuan, Y., Wang, Z., Guo, L., Liu, H.: Barrier lyapunov functions-based adaptive fault tolerant control for flexible hypersonic flight vehicles with full state constraints. IEEE Transactions on Systems, Man, and Cybernetics: Systems. 50(9), 3391-3400 (2020)

4. Kristic, M., Kanellakopoulos, I., Kokotovic, P.: Nonlinear and adaptive control design. New York, USA: Wiley. (1995)

5. Wang, M., Wang, C.: Neural learning control of purefeedback nonlinear systems. Nonlinear Dynamics. 79(4), 2589-2608 (2015)

6. Zhou, Q., Zhao, S., Li, H., Lu, R., Wu, C.: Adaptive neural network tracking control for robotic manipulators with dead zone. IEEE Transactions on Neural Networks and Learning Systems. 30(12), 3611-3620 (2019)

7. Liu, D., Liu, Z., Chen, C.L.P., Zhang, Y.: Distributed adaptive neural control for uncertain multi-agent systems with unknown actuator failures and unknown dead zones. Nonlinear Dynamics. 99(2), 1001-1017 (2020)

8. Yu, X., Wang, T., Qiu, J., Gao, H.: Barrier lyapunov function-based adaptive fault-tolerant control for a class of strict-feedback stochastic nonlinear systems. IEEE Transactions on Cybernetics. 51(2), 938-946 (2021)

9. Tong, S., Min, X., Li, Y.: Observer-based adaptive fuzzy tracking control for strict-feedback nonlinear systems with unknown control gain functions. IEEE Transactions on Cybernetics. 50(9), 3903-3913 (2020)
10. Wang, M., Wang, C.: Learning from adaptive neural dynamic surface control of strict-feedback systems. IEEE Transactions on Neural Networks. 26(6), 1247-1259 (2015)

11. Zhao, K., Song, Y.: Removing the feasibility conditions imposed on tracking control designs for state-constrained strict-feedback systems. IEEE Transactions on Automatic Control. 64(3), 1265-1272 (2019)

12. Chen, M., Ge, S.S.: Adaptive neural output feedback control of uncertain nonlinear systems with unknown hysteresis using disturbance observers. IEEE Transactions on Industrial Electronics. 62(12), 7706-7716 (2015)

13. Bechlioulis, C.P., Rovithakis, G.A.: Robust adaptive control of feedback linearizable MIMO nonlinear systems with prescribed performance. IEEE Transactions on Automatic Control. 53(9), 2090-2099 (2008)

14. Hua, C., Liu, G., Li, L., Guan, X.: Adaptive fuzzy prescribed performance control for nonlinear switched timedelay systems with unmodeled dynamics. IEEE Transactions on Fuzzy Systems. 26(4), 1934-1945 (2018)

15. Yang, Y., Tan, J., Yue, D.: Prescribed performance tracking control of a class of uncertain pure-feedback nonlinear systems with input saturation. IEEE Transactions on Systems, Man, and Cybernetics: Systems. 50(5), 17331745 (2020)

16. Zhang, L., Yang, G-H.: Adaptive fuzzy prescribed performance control of nonlinear systems with hysteretic actuator nonlinearity and faults. IEEE Transactions on Systems, Man, and Cybernetics: Systems. 48(12), 2349-2358 (2018)

17. Bikas, L.N., Rovithakis, G.A.: Combining prescribed tracking performance and controller simplicity for a class of uncertain MIMO nonlinear systems with input quantization. IEEE Transactions on Automatic Control. 64(3), 1228-1235 (2019)

18. Dai, S-L., Wang, M., Wang, C.: Neural learning control of marine surface vessels with guaranteed transient tracking performance. IEEE Transactions on Industrial Electronics. 63(3), 1717-1727 (2016)

19. Wang, M., Yang, A.: Dynamic learning from adaptive neural control of robot manipulators with prescribed performance. IEEE Transactions on Systems, Man, and Cybernetics: Systems. 47(8), 2244-2255 (2017)

20. Liu, Y., Liu, X., Jing, Y.: Adaptive neural networks finitetime tracking control for non-strict feedback systems via prescribed performance. Information Sciences. 468, 29$46(2018)$

21. Liu, Y., Liu, X., Jing, Y., Chen, X., Qiu, J.: Direct adaptive preassigned finite-time control with time-delay and quantized input using neural network. IEEE Transactions on Neural Networks and Learning Systems. 31(4), 12221231 (2020)

22. Wang, H., Bai, W., Zhao, X., Liu, X.P.: Finite-timeprescribed performance-based adaptive fuzzy control for strict-feedback nonlinear systems with dynamic uncertainty and actuator faults. IEEE Transactions on Cybernetics. doi: 10.1109/TCYB.2020.3046316 (2020)

23. Zhou, X., Gao, C., Li, Z-G., Ouyang, X-Y., Wu, LB.: Observer-based adaptive fuzzy finite-time prescribed performance tracking control for strict-feedback systems with input dead-zone and saturation. Nonlinear Dynamics. 103(2), 1645-1661 (2021)

24. Cao, Y., Song, Y.: Performance guaranteed consensus tracking control of nonlinear multiagent systems: A finite-time function-based approach. IEEE Transactions on Neural Networks and Learning Systems. 32(4), 1536$1546(2021)$ 
25. Nussbaum, R.D: Some remarks on a conjecture in parameter adaptive control. Systems \& Control Letters. 3(5), 243-246 (1983)

26. Wu, Y., Xie, X-J.: Robust adaptive control for stateconstrained nonlinear systems with input saturation and unknown control direction. IEEE Transactions on Systems, Man, and Cybernetics: Systems. 51(2), 1192-1202 (2021)

27. Wang, L., Chen, C.L.P.: Adaptive fuzzy dynamic surface control of nonlinear constrained systems with unknown virtual control coefficients. IEEE Transactions on Fuzzy Systems. 28(8), 1737-1747 (2020)

28. Gao, H., Chen, T., Lam, J.: A new delay system approach to network-based control. Automatica. 44(1), 39$52(2008)$

29. Liu, A., Zhang, W., Yu, L., Liu, S., Chen, M.Z.Q.: New results on stabilization of networked control systems with packet disordering. Automatica. 52, 255-259 (2015)

30. Tabuada, P.: Event-triggered real-time scheduling of stabilizing control tasks. IEEE Transactions on Automatic Control. 52(9), 1680-1685 (2007)

31. Wang, M., Wang, Z., Chen, Y., Sheng, W.: Adaptive neural event-triggered control for discrete-time strictfeedback nonlinear systems. IEEE Transactions on Cybernetics. 50(7), 2946-2958 (2020)

32. Ling, S., Wang, H., Liu, X.P.: Fixed-time adaptive eventtriggered tracking control of uncertain nonlinear systems. Nonlinear Dynamics. 100(4), 3381-3397 (2020)

33. Xing, L., Wen, C., Liu, Z., Sun, H., Cai, J.: Eventtriggered adaptive control for a class of uncertain nonlinear systems. IEEE Transactions on Automatic Control. 62(4), 2071-2076 (2017)

34. Qiu, J., Sun, K., Wang, T., Gao, H.: Observer-based fuzzy adaptive event-triggered control for pure-feedback nonlinear systems with prescribed performance. IEEE Transactions on Fuzzy Systems. 27(11), 2152-2162 (2019)

35. Wang, M., Wang, Z., Chen, Y., Sheng, W.: Eventbased adaptive neural tracking control for discrete-time stochastic nonlinear systems: A triggering threshold compensation strategy. IEEE Transactions on Neural Networks and Learning Systems. 31(6), 1968-1981 (2020)

36. Cao, L., Li, H., Dong, G., Lu, R.: Event-triggered control for multiagent systems with sensor faults and input saturation. IEEE Transactions on Systems, Man, and Cybernetics. 51(6), 3855-3866 (2021)

37. Xing, L., Wen, C., Liu, Z., Sun, H., Cai, J.: Eventtriggered output feedback control for a class of uncertain nonlinear systems. IEEE Transactions on Automatic Control. 64(1), 290-297 (2019)

38. Zhang, T., Ge, S.S.: Adaptive neural control of MIMO nonlinear state time-varying delay systems with unknown dead-zones and gain signs. Automatica. 43(6), 1021-1033 (2007)

39. Sanner, R.M., Slotine, J-J. E.: Gaussian networks for direct adaptive control. IEEE Transactions on Neural Networks. 3(6), 837-863 (1992)

40. Levant, A.: Robust exact differentiation via sliding mode technique. Automatica. 34(3), 379-384 (1998)

41. Levant, A.: Higher-order sliding modes, differentiation and output-feedback control. International Journal of Control. 76(9-10), 924-941 (2003)

42. Yu, J., Shi, Peng., Zhao, Lin.: Finite-time command filtered backstepping control for a class of nonlinear systems. Automatica. 92, 173-180 (2018)

43. Polycarpou, M.M., Ioannou, P.A.: A robust adaptive nonlinear control design. Automatica. 32(3), 423-427 (1996)
44. Ryan, E.P.: A universal adaptive stabilizer for a class of nonlinear systems. Systems \& Control Letters. 16(3), 209-218 (1991)

45. Li, D., Li, D.: Adaptive tracking control for nonlinear time-varying delay systems with full state constraints and unknown control coefficients. Automatica. 93, 444-453 (2018) 